\title{
A Guide to Doing Statistics in Second Language Research Using SPSS
}

\author{
Jenifer Larson-Hall. \\ New York and London: Routledge, 2010. 423 pages. ISBN 10: 0-8058-6185-8.
}

When Larson-Hall wrote this book, she had in mind, those second language researchers who feel a little uncomfortable when dealing with statistics. With this introduction to statistics through the Statistical Package for the Social Sciences (SPSS) she found a suitable way to help researchers first to interpret the statistics tests and then to learn how to generate descriptive statistics, choose a statistical test, and conduct and interpret the basic tests that a researcher may need.

In A Guide to Doing Statistics in Second Language Research Using SPSS, LarsonHall mainly draws her data sets from real Second Language Acquisition studies, and these are featured in a companion website (http://www.routledge.com/textbooks/9780805861853) so that readers can use raw data to complete the exercises contained in the book. Thanks to these sets the author allows the reader to access and to work on other researchers' data. Although at the beginning she considered working with the statistical program " $R$ " and with the SPSS, she decided to make things easier and to focus on the SPSS as the R program, although freeware, is more difficult to cope with. Thus, the main issues of this book are illustrated with no few SPSS windows, tables, figures and the proposal of a series of exercises and activities whose answers are found in the abovementioned website.

The book is meant to be read in a chronological order. Part I presents fundamental concepts in statistics and Part II provides information about statistical tests that are commonly used in second language research.

Part I focuses on a number of issues such as the entering of data and how to manipulate them, the identification of variables and the null hypothesis testing, or the author's preference for the use of the term "statistical" rather than "statistically significant. Particular attention is paid to:

1. Her option for the broad division of the level of measurement into continuous and categorical variables rather than the narrow 
categorisation of the traditional measurement scales.

2. The role of the researcher, as it is up to him/her to consider one variable as continuous or categorical according to his interests.

3. The function of variables in research, since the statistic technique chosen will depend on the sort of variable we deal with.

4. Some proposals in reporting research articles. The author stands for exact $p$-values (unless they are quite small), the use of confidence intervals, and the effect sizes to avoid significant drawbacks in studies with small sample sizes.

5. The issue of power is taken seriously when she suggests the use of Cohen's (1988) guidelines to understand that power may frequently be the real reason why the expected results were not found. Such is her concern about power that she provides a useful table using $\mathrm{R}$ commands to calculate power test in ANOVA, Correlation, Ttest and Chi-square.

6. Her preference for raising the $\alpha$ level to 0.10 instead of the current 0.05, on the grounds of a better balance between Type I and Type II errors.

In Part II the author starts by introducing and giving an overview of the statistical tests that will be covered in the book: correlation, introduction to multiple regression, chi-square, t-tests, one way analysis of variance, ANOVA with more than one independent variance or with repeated measures, analysis with covariates and some non-parametric tests. Her intention in this second part is to move from what can be considered the easier statistic tests to the more difficult ones. Larson-Hall advances the criteria that should be considered in the choice of a statistical test:

- whether you are trying to test a relationship between variables or look for group differences,

- how many variables you have and how many levels or groups there are within the variable,

- whether the variables can be considered continuous or categorical,

- whether the variables are seen as independent or dependent (or not really either). 
She begins with correlation and shows through several SPSS windows how to create scatterplots, how to add a regression line and how to get multiple scatterplots. Then, after some comments on the assumptions required, she focuses on the effect size for correlation. Finally she goes through the Pearson's r or Spearman's rho and the Cronbach's alpha. Regression is the next statistic. The explanatory and the predictive models are commented. Besides the $\mathrm{R}^{2}$ the author suggests that researchers should provide information about the "R square Change". She prefers to use the sequential regression rather than the stepwise regression because in so doing it is the researcher and not the computer who decides which term to eliminate. She also discusses the variety of "rules of thumb" for the number of samples required in a regression analysis.

Then she moves to the Chi-square use to calculate the difference between the scores you observe and the scores you would expect and then to see if the magnitude of the difference is large or small on the chi-square distribution. She discusses the test for goodness of fit of the data and the test for group independence.

As regards group differences in parametric tests, Larson-Hall begins with the independent-samples t-test and the paired-samples t-test. Besides the Bonferroni adjustment she emphasises the importance of the effect sizes over $p$-values in looking at t-tests results, since $p$-values are greatly influenced by sample sizes while effect sizes are not. She shows how to calculate the effect size.

The one-way ANOVA, which is used when there are three or more groups and there is only a dependent variable and only one independent variable. As in no few cases the assumption of normal distribution is not fulfilled she points out that either a robust test or a non-parametric test is required. Her analysis about the omnibus test and post-hocs or planned comparisons should be mentioned. Help is also provided to obtain the effect size.

The analysis of variance is extended to the two-way ANOVA, that is, when there are two independent variables. In this design, not only the main effects of the variables but also the interaction between the independent variables must be considered. Due to complexity she recommends not to use more than three independent variables.

In the Repeated-Measures ANOVA, besides the assumptions of other parametric tests, comments on sphericity are added. It is suggested using the correction factor with sphericity and the Games-Howell post-hoc if the 
heterogeneity of variances requirements is violated. When the independent variables are continuous the analysis of covariance (ANCOVA) is required. She recommends using this statistic with caution as it contains even more assumptions than a regular ANOVA.

In the last chapter the following non-parametric statistics are considered: the binomial test, the Mann-Whitney Test, the Kruskal-Wallis Test, the Wilcoxon Signed Ranks Test, the MacNemar Test and the Friedman Test. On the grounds that there are still authors that despite some violations of assumptions in parametric tests prefer using parametric tests to nonparametric tests, the author, as does Howell (2002), would rather be using robust statistical methods. It is believed that these methods will shortly overtake what are now the most common nonparametric tests, and may eventually overtake the traditional parametric tests.

On the whole A Guide to Doing Statistics in Second Language Research Using SPSS deserves to be taken into account if you feel a bit helpless at statistics and you are involved in second language research - as the case for LSP studies may be. This book supplies the background you may need to conduct and interpret the basic statistical tests. Worth highlighting are the following issues:

- The use the author makes of the SPPS windows, tables and figures to show how to manage data.

- The application activities at the end of each chapter.

- The author's little concern with formulae.

- The importance of showing the effect size of each statistic.

- Her insistence on showing how to report results in our research.

- The importance of the Website recommended.

- The great help you may find in the glossary.

One might question the established order with regard to Correlation, Regression or Chi-square chapters in the display of the book and the use now and then of the $\mathrm{R}$ statistical package, but $\mathrm{I}$ think that the author certainly succeeds in filling in the existing gaps readers may have in second language research. 
Reviewed by Honesto Herrera Soler

Universidad Complutense de Madrid (Spain)

herrera@ffilol.ucm.es

\section{REFERENCES}

Cohen, J. (1988). Statistical Power Analysis for the Behavioural Sciences. Newbury Park, CA: Sage.
Howell, D.C. (2002). Statistical Methods for Psychology. Pacific Grove, CA: Duxbury/Thomson Learning. 
